\title{
Avaliação Automática do Alinhamento Ocular para Detecção do Estrabismo Usando Redes YOLO e U-net
}

\author{
Thayane de Oliveira Simões* \\ João Dallyson Sousa de Almeida* Aristófanes Correa Silva* \\ Anselmo Cardoso de Paiva* \\ * Núcleo de Computação Aplicada - Universidade Federal do Maranhão \\ São Luís, MA, Brasil \\ (e-mail: \{thayanesimoes19@gmail.com, jdallyson@nca.ufma.br, \\ ari@dee.ufma.brpaiva@nca.ufma.br,\}
}

\begin{abstract}
Strabismus is an ophthalmological disease characterized by an imbalance in the ocular muscles, so that the two eyes do not fix the same point or object at the same time. Its incidence can occur from the first months of life to adulthood. Late detection may cause double vision or loss of vision, hence the importance of early detection of strabismus for its prognosis and effective treatment. That said, in this work is proposed a new method that uses neural networks to automatically detect strabismus by evaluating the positioning of the limbus center in relation to the center of the corners of the eyes. To do so, we use techniques of image processing and machine learning, obtaining sensitivity of $66,7 \%$, specificity of $60 \%$ and accuracy of $65,6 \%$.

Resumo: O estrabismo é uma doença oftalmológica caracterizada por um desequilíbrio nos músculos oculares, fazendo com que os dois olhos não fixem o mesmo ponto ou objeto ao mesmo tempo. Sua incidência pode ocorrer desde os primeiros meses de vida até a idade adulta. A detecção tardia pode causar visão dupla ou perda da visão, por isso a importância da deteç̧ão precoce do estrabismo para seu prognóstico e tratamento eficaz. Dito isso, neste trabalho é proposto um novo método que utiliza redes neurais para detectar automaticamente o estrabismo avaliando o posicionamento do centro do limbo em relação ao centro dos cantos dos olhos. Para tanto, utiliza-se técnicas de processamento de imagens e aprendizado de máquina, obtendo sensibilidade de $66,7 \%$, especificidade de $60 \%$ e acurácia de $65,6 \%$.
\end{abstract}

Keywords: Strabismus; Hirschberg test; Segmentation; YOLOv3; U-net ;Detection.

Palavras-chaves: Estrabismo; Teste de Hirschberg; Segmentação; YOLOv3; U-net; Detecção.

\section{INTRODUÇÃO}

O estrabismo é uma patologia oftalmológica comum que possui uma prevalência relativamente alta de cerca de $4 \%$ da população. É caracteizado pelo desvio do alinhamento binocular, no qual um ou ambos os olhos podem se voltar para dentro, para fora, para cima ou para baixo (Zheng et al., 2018). É uma doença que abrange as situações nas quais o cérebro identifica duas imagens com diferentes focos, em vez de duas imagens que se concentram no mesmo objeto.

Por ser uma doença caracterizada por uma alteração ocular que desalinha os olhos em diferentes posições, o estrabismo deve ser diagnosticado nos primeiros anos de vida para que seu tratamento seja eficaz e evite consequências como baixa visão podendo progredir para a cegueira. Por sua vez, o estrabismo pode ser detectado através do teste de acuidade visual, cover test e do teste de Hirschberg que consiste em incidir um pequeno foco de luz nos olhos do paciente, observando se o reflexo em cada olho está localizado no mesmo lugar em cada uma das córneas, sendo que este é o mais utilizado para detectar essa patologia (Almeida et al., 2012). Por isso, a detecção precoce e o tratamento adequado aumentam a probabilidade do alinhamento ser restaurado ao normal.

Dessa forma, sistemas de detecção e diagnóstico auxiliado por computador (CAD - Computer-Aided Detection e $C A D x$ - Computer-Aided Diagnosis) têm sido propostos com o objetivo de auxiliar o especialista e identificar anormalias mascaradas. Esses sistemas têm sido desenvolvidos por inúmeros grupos de pesquisa, visando auxiliar, por exemplo, a detecção e o diagnóstico precoce de algumas doenças como o câncer. Assim, essa categoria de sistema vem ganhando cada vez mais espaçoo na medicina moderna (ALMEIDA et al., 2013).

Diferentemente das abordagens tradicionais, exames de cobertura e teste de Hirschberg, este trabalho propõe uma nova abordagem computacional para detectar a presença de estrabismo utilizando fotografia da face, na qual analisa-se automaticamente a diferença entre o centro do limbo em relação ao centro dos cantos dos olhos, utilizando técnicas de processamento de imagens e inteligência 
artificial, a fim de oferecer uma segunda opinião aos especialistas, servindo como apoio para decisões.

\section{TRABALHOS RELACIONADOS}

Na literatura existem algumas pesquisas no contexto desse trabalho. Abaixo enumera-se alguns deles.

Loudon et al. (2011) propuseram uma metodologia de detecção de estrabismo e ambliopia em crianças utilizando um Scanner de Visão Pediátrica (PVS), identificando a fixação ocular em ambos os olhos simultaneamente, onde o objetivo do trabalho foi a investigação da possibilidade de utilização do PVS para detectar o estrabismo. O teste foi realizado em crianças recrutadas no Departamento de Oftalmologia do Hospital Infantil de Boston, no qual a base contém 64 imagens de ambliopia e 66 imagens de estrabismo e todas realizaram exames de rotina. Com isso, realizou-se uma comparação entre o PVS e os exames oftalmológicos para comprovar a eficiência do software, obtendo $60 \%$ de especificidade e $96 \%$ de sensibilidade.

Em Almeida et al. (2012) apresenta-se uma metodologia para auxiliar no diagnóstico de estrabismo em imagens digitais através do teste de Hirschberg, a base utilizada contém 40 pacientes estrábicos. Inicialmente, delimitaram a região dos olhos através de projeções horizontais. Em seguida, realizaram a localização dos olhos utilizando descritores de textura baseados em funções geoestatísticas e Máquina de Vetor de Suporte (SVM). Para localizar o centro do limbo desenvolveram um algoritmo adaptado da transforamda de Hough. Por fim, localizaram as coordenadas do brilho e verificaram o alinhamento dos olhos. Essa abordagem obteve resultados na faixa de $100 \%$ de sensibilidade, $91,3 \%$ de especificidade e $94 \%$ de acurácia.

Lu et al. (2018) apresentaram uma estrutura end-to-end chamada RF-CNN para alcançar a detecção automatizada de estrabismo aplicado na telemedicina, que significa fazer uso da tecnologia de telecomunicações e informática para oferecer assistência médica à distância. Os testes foram realizados no banco de dados de tele-estrabismo no qual foi rotulado e coletado por oftalmologistas, a detecção é composta por (1) segmentação da região dos olhos, utilizando a rede neural R-FCN e (2) classificação do estrabismo, utilizando uma rede neural profunda. O método proposto obteve sensibilidade de $93,3 \%$, especificidade de $96,1 \%$, precisão igual a 93,8\% e AUC igual a $98,6 \%$.

Já Chen et al. (2018), desenvolveram uma metodologia para reconhecer o estrabismo utilizando dados de rastreamento ocular e seis modelos de redes neurais convolucionais diferentes. Utilizaram imagens de desvio do olhar (GaDe) para caracterizar e obter os dados de rastreamento ocular e suas devidas precisões dos pontos do olhar, no qual possui 17 casos de estrabismo. Essas imagens servem como entrada para as redes neurais convolucionais que foram treinadas usando o banco de dados ImageNet, a saída da rede é utilizada para extrair características para serem classificadas em nomais ou estrábicas pelo SVM, essa abordagem obtiveram o melhor resultado com a rede VGG-S com especificidade de 96\%, sensibilidade de $94,1 \%$ e uma precisão de $95,2 \%$.

Estes são exemplos de metodologias que foram desenvolvidas para detecção do estrabismo. Três pontos importantes comuns nestes trabalhos são à necessidade de uma fase de localização dos olhos, a localização do limbo (estrutura de transição entre a córnea e a esclera) e a metologia empregada em cada trabalho limita-se apenas ao exame em estudo. Em nosso método tentar-se-á explorar essas deficiências com o intuito de melhorar as métricas que avaliam o desempenho.

\section{METODOLOGIA PROPOSTA}

A metodologia proposta consiste nas seguintes etapas: aquisição da base de imagens, localização do limbo e çocalização dos olhos, segmentação da esclera, localização dos cantos dos olhos e por fim a classificação em estrábico ou normal. A Figura 1 ilustra as etapas da metodologia.

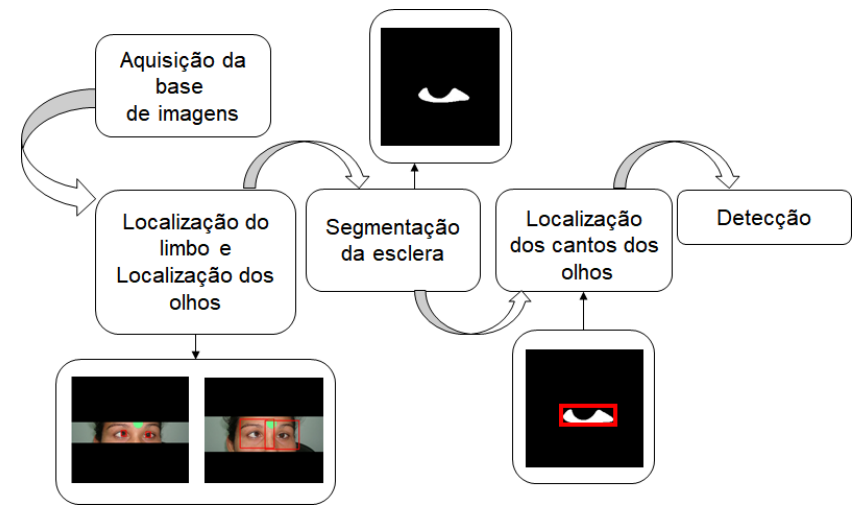

Figura 1. Metodologia proposta.

\subsection{Bases de imagens}

Este trabalho utiliza as bases de imagens UBIRIS.v2 (Proença and Alexandre, 2005), a base privada SSRBC 2017 (Das et al., 2014), a base privada de Teste do Brückner utilizada por (Silva et al., 2018) e a base privada de estrabismo utilizada por (De Almeida et al., 2015).

A base privada do Teste de Brückner foi utilizada para a realização do treinamento da rede Yolo na etapa de localização dos olhos. Na qual, contém no total 58 imagens divididas em duas classes: pacientes normais (33 imagens) e pacientes com patologia (25 imagens) (Silva et al., 2018).

As bases de imagens utilizadas para realizar o treinamento da rede U-net para realizar a segmentação da esclera foram a UBIRIS.v2 e SSRBC 2017. A base UBIRIS.v2 contém 201 imagens com 400 pixels de largura e 300 pixels de altura, enquanto que o conjunto de imagens do SSRBC 2017 possui 120 imagens oculares de 30 indivíduos.

Já nos experimentos, foram utilizadas as imagens da base privada de estrabismo que foram adquiridas em um consultório oftalmológico em São Luís-MA, contendo 45 pacientes, sendo que cada paciente contém 5 posições diferentes: posição primária do olhar (PPO), posição do olhar direcionado para esquerda (LEVO), posição do olhar direcionado para direita (DEXTRO), posição do olhar direcionado para baixo (INFRA) e posição do olhar direcionado para cima (SUPRA) (De Almeida et al., 2015). A Figura 2 apresenta um exemplo da base.

A base contém 225 imagens divididas em duas classes: 40 pacientes com estrabismos e 5 pacientes normais e para 
cada paciente possuem marcações da esclera, marcações do limbo e o diagnóstico do especialista.

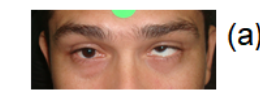

(b)
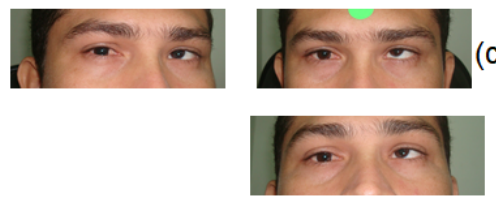

c) (e)
Figura 2. Imagens nas 5 posições do olhar: (a) SUPRA, (b) DEXTRO, (c) PPO, (d) LEVO, (e) INFRA (De Almeida et al., 2015).

\subsection{Localização do limbo e Localização dos olhos}

Nesta etapa para localizar as regiões dos olhos e do limbo, que é a região dos olhos que separa a esclera (região branca) da íris, foi utilizada a rede YOLO que possui uma aquitetura de rede neural para detecção de obejtos. Proposta por (Redmon et al., 2016) a YOLO trata a detecção de objetos como um poblema de regressão, cujo resultado é a delimitação de bounding boxes.

Neste trabalho, utilizou-se a YOLOv3 proposta por (Redmon and Farhadi, 2018), sua característica é o conceito de âncoras que estão ligados à predição e sua arquitetura contém blocos de convolução e blocos residuais. O primeiro bloco de convolução é chamado de DarkConv, que serve como entrada de kernel 3x3 para o segundo bloco DarkBlock, no qual o resultado é duplicado, o terceiro bloco LastLayers corresponde a camada final da rede composto por 5 blocos DarkConv e o bloco final da YOLOv3 é chamada de YOLO Output que tem como entrada a saída do bloco LastLayers e é reponsavel por prover a saída da rede (SILVA et al., 2019).

A base de treinamento nesta etapa foi a (Silva et al., 2018) e por conter poucas amostras foi utilizada a técnica Data Augmentation resultando em 1428 imagens. Sua arquiteura foi construída com a função de ativação LeakyReLU, convoluções de 3x3 com 256 filtros e foram definidas 100 épocas.

\subsection{Segmentação da esclera}

A esclera é a região externa branca e opaca dos olhos e apresenta vasos sanguíneos que podem ser usados no reconhecimento biométrico. A segmentação da esclera é a primeira etapa para o reconhecimento ocular, seu desempenho depende da iluminação e das múltiplas direções do olhar que influenciam diretamente no resultado esperado (Maheshan et al., 2018).

Para a segmentação da esclera utiliza-se a arquitetura da U-net. Essa é uma rede totalmente convolucional que é um modelo para segmentação de imagens biomédicas que tem como principal característica a utilização de poucas imagens no treinamento. Sua arquitetura consiste em um caminho de contratação para capturar o contexto e um caminho de expansão simétrico que permite uma segmentação mais precisa (Ronneberger et al., 2015).
Assim, as bases UBIRIS.v2 e SSRBC 2017 foram utilizadas como conjunto de treinamento da rede, pois são bases que possuem apenas a região dos olhos.

\subsection{Localização dos cantos dos olhos}

Após completar os estágios anteriores, em seguida localizamos os cantos dos olhos, utilizando uma bounding box na esclera segmentada, esta é uma técnica que identifica o menor retângulo presente em um determinado objeto. No entanto, há casos em que não foi possível detectar os cantos dos olhos utilizando a esclera como referência, devido a segmentação impecisa, então nessas circunstâncias o limbo é utilizado como referência, para isso verifica se as coordenadas do limbo é menor (para região da direita) ou maior (para região da esquerda) que a as coordenadas do canto do olhos. Nas Figuras 3 (b) e (c) a localização do limbo e dos cantos dos olhos, podem ser observados respectivamente.

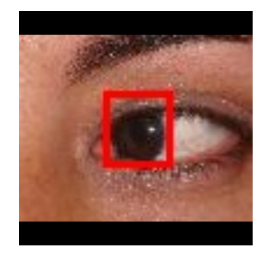

(a)

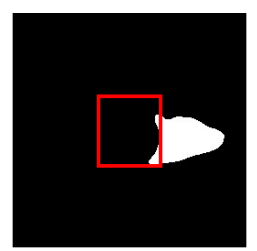

(b)

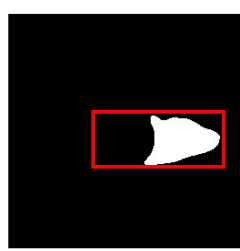

(c)
Figura 3. (a) Imagem original, (b) Localização do limbo, (c) Localização dos cantos dos olhos (De Almeida et al., 2015).

\subsection{Deteç̧̃̃o do Estrabismo}

Para a realização dos experimentos foram utilizadas imagens adquiridas do teste de Hirschberg nas cinco posições do olhar DEXTRO, INFRA, LEVO, PPO e SUPRA, que é um teste que analisa o reflexo da luz na córnea. Neste teste, é necessária uma taxa de conversão para obter o ângulo estrábico (em dioptrias de prisma $\Delta$ ) a partir do deslocamento observado no reflexo da córnea (em milímetros) (Hasebe et al., 1998). No entanto, para detectar o estrabismo o método proposto utiliza o centro dos cantos dos olhos e o centro do limbo como parâmetros de análise do alinhamento dos olhos.

Nesse teste, um olho é o fixador e o outro olho é o que será analisado. Uma referência que deve ser considerada é o ângulo kappa, que é gerado devido ao desalinhamento entre os eixos visual e pupilar (Bicas, 2009).

A magnitude do alinhamento em cada um dos olhos, é obtida medindo a distância do centro dos cantos dos olhos para o centro do limbo na horizontal, denominado Desvio Horizontal (DH), isso é expresso pela Equação 1:

$$
D H p=\left|x_{c}-x_{l}\right|
$$

onde $x c$ e $x l$ são respectivamente coordenadas do centro dos cantos dos olhos e centro do limbo de cada olho em pixels e $D H p$ é o valor do desvio horizontal em pixels.

Em seguida, utiliza-se o valor médio do diâmetro horizontal do limbo, onde realizou-se a conversão de pixel para milímetro (pixelMM), tomando como referência o valor 
médio do diâmetro do limbo de um adulto igual a $11 \mathrm{~mm}$ (Khng and Osher, 2008), como apresentam as Equações 2 e 3 .

$$
\begin{gathered}
\text { pixel } M M=11 / D L \\
D H m m=D H * \operatorname{pixel} M M
\end{gathered}
$$

onde $D L$ e $D H m m$ são respectivamente diâmetro do limdo de um adulto e desvio horizontal em milímetros.

Por fim, o valor do desvio é convertido para dioptrias prismáticas ( $\Delta$ - métrica utilizada pelo especialista). Esse cálculo é expresso pela seguinte Equação 4.

$$
D H \Delta=D P * D H m m
$$

onde $D P$ representa uma constate igual a $15 \Delta$ (Schwartz, 2006).

A Figura 4 demonstra um exemplo do desalinhamento em um paciente, na qual é possível perceber o desvio horizontal entre o centro do limbo (ponto vermelho) e o centro dos cantos dos olhos (ponto verde).

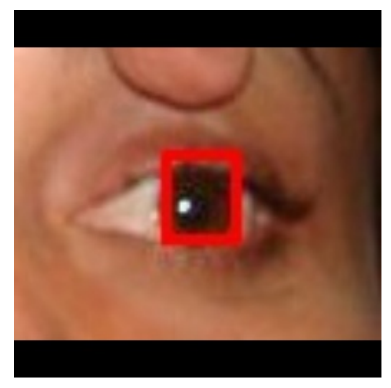

(a)

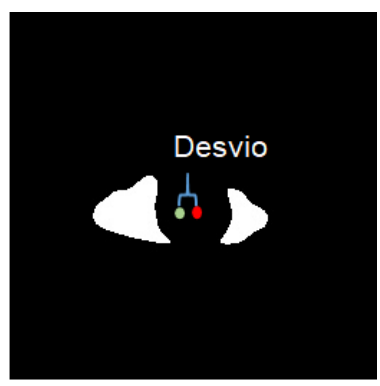

(b)
Figura 4. (a) Localização do limbo, (b) Localização dos centros, onde o ponto verde é referente a centro dos cantos dos olhos e o ponto vermelho é o centro do limbo.

\section{RESULTADOS E DISCUSSÕES}

Esta seção apresenta e discute os resultados alcançados utilizando a metodologia proposta. Serão utilizadas as métricas sensibilidade, especificidade, acurácia e dice (Dice, 1945) para validar os experimentos realizados.

$\mathrm{Na}$ etapa de localização dos olhos a rede YOLOv3 foi treinada com 1186 imagens e validada em 225 imagens. A rede foi treinada para identificar a região do limbo, após isso uma nova foi bounding box foi criada para localizar os olhos a partir do centro da bounding box do limbo, como apresenta a Figura 5. No entanto, há casos em que apenas uma região do limbo foi encontrado, assim as coordenadas da região encontrada foram espelhadas, como apresenta a Equação 5. Com essa abordagem obtivemos 97,3\% de sensibilidade, $99,5 \%$ de especificidade e $98,4 \%$ de acurácia para a localização do limbo.

$$
\begin{gathered}
x \min =\mid(x \min -x \text { MirrorAxis }) \mid+x \text { Mirror Axis } \\
x \max =\mid(x \max -x \text { MirrorAxis }) \mid+x \text { Mirror Axis }
\end{gathered}
$$

onde $x \min$ e $x \max$ representam respectivamente o menor e maior valor da coordenada x e xMirrorAxis a coluna da imagem original divida por 2 .
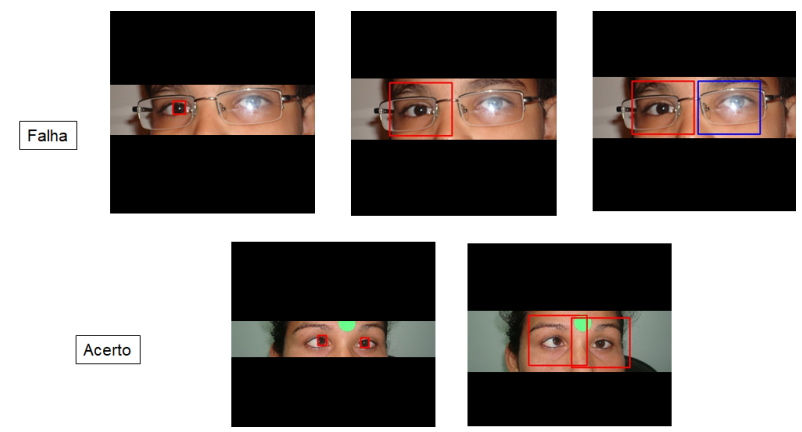

Figura 5. Resultado da rede YOLOv3 em vermelho e espelhamento representada pela cor azul.

A Figura 5 apresenta um caso de falha que como pode ser observado o erro acontence em imagens que possuem flash e um caso de acerto.

A partir da Figura 5 observa-se que após o espelhamento foi possível identicar os dois olhos, enquanto que a localização do limbo não foi tão precisa.

Para a segmentação da esclera, utilizou-se 320 imagens das (Proença and Alexandre, 2005) e (Das et al., 2014), a rede foi construída com a função de ativação Leaky ReLU, convoluções de 3x3, foram definidas 5 épocas e 700 interações. Com a rede treinada a mesma foi utilizada para segmentar as 450 imagens, sendo 225 dos olhos direito e 225 dos olhos esquerdo dos pacientes.

A Figura 6 apresenta os resultados da segmentação da esclera utilizando a rede U-net, onde como pode ser observado na Figura 6 (b) possuem falsos positivos (regiões não pertencentes a esclera), por isso foram selecionados os maior contornos da imagem para preenchimento de buracos e remover os falsos positivos, como apresenta a Figura $6(\mathrm{c})$.

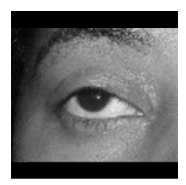

(a)

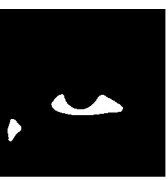

(b)

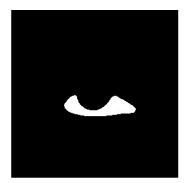

(c)

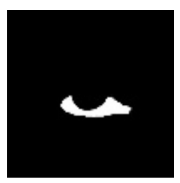

(d)
Figura 6. (a) - Imagem Original, (b) Resultado da segmentação, (c) Pós-processamento selecionando os maiores contornos , (d) Marcação.

Com essa abordagem obtivemos dice de 77,1\%, especificidade de $99 \%$, sensibilidade de $80 \%$ e acurácia de $98,5 \%$. Com isso, para a etapa posterior foram consideras apenas 320 imagens que resultaram em pelo menos uma área da esclera segmentada, pois com sua ausência não é possível localizar os cantos dos olhos.

Em seguida, com o centro dos cantos dos olhos localizados, nos testes realizados o método obteve uma taxa de acerto de $87,2 \%$ na localização dos cantos dos olhos. 
Ao analisar a detecção de estrabismo, os estudos de (Choi and Kushner, 1998) estimaram desvios com erro médio de $5 \Delta$ a $10 \Delta$. Portanto, a partir dos resultados encontrados utilizou-se a tolerância ao erro de $\pm 10 \Delta$ para avaliar o valor estimado pelo método em relação à medida do especialista. Os pacientes identificados pelo especialista com $0 \Delta$ são aqueles que não possuem estrabismo e os demais são os que possuem a patologia. Após as etapas de localização do centro do limbo e localização do centro dos cantos dos olhos, apenas 32 pacientes (27 estrábiscos e 5 normais) foram levados em consideração nos testes, devido ao erro na segmentação da esclera no qual não foi possível identicar o centro dos cantos dos olhos. As Figuras 7, 8, 9, 10 e 11 apresentam os gráficos para as imagens de cada posição do olhar comparando o método proposto com a medida do desvio realizada pelo especialista baseado no teste de Hirschberg.

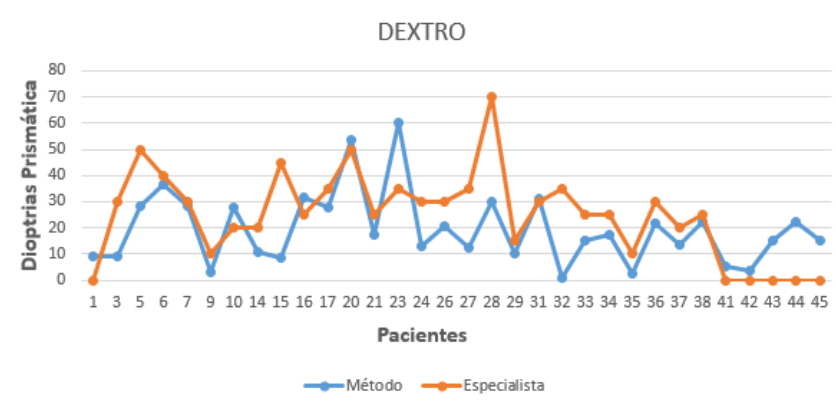

Figura 7. Resultado do método proposto na posição DEXTRO em comparação com o diagnóstico do especilista.

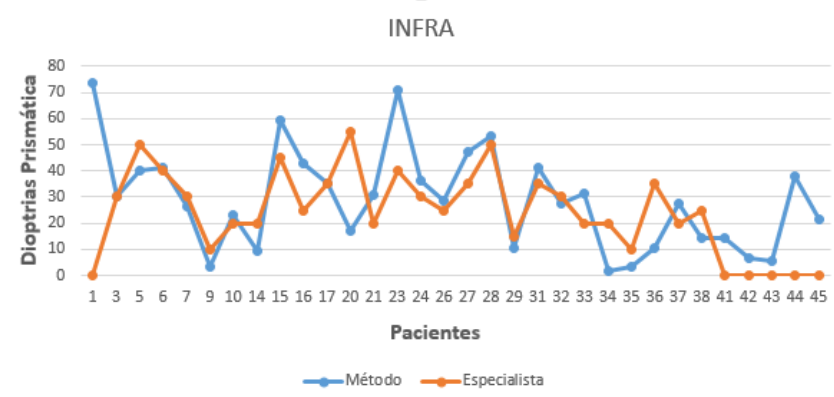

Figura 8. Resultado do método proposto na posição INFRA em comparação com o diagnóstico do especilista.

LEVO

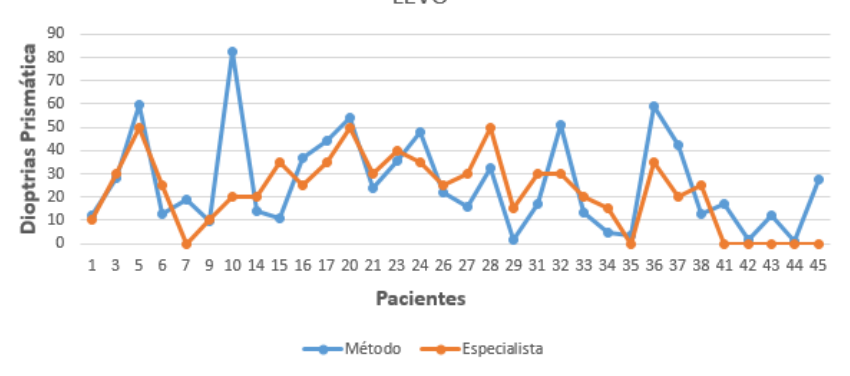

Figura 9. Resultado do método proposto na posição LEVO em comparação com o diagnóstico do especilista.

A partir das Figuras 7, 8, 9, 10 e 11 pode-se inferir que o método proposto apresenta de forma geral bons resultados, resaltando que os casos de erros são referentes a localização

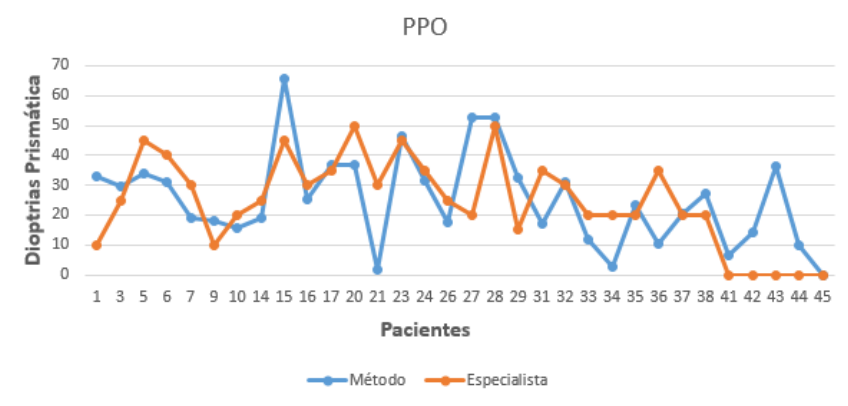

Figura 10. Resultado do método proposto na posição PPO em comparação com o diagnóstico do especilista.

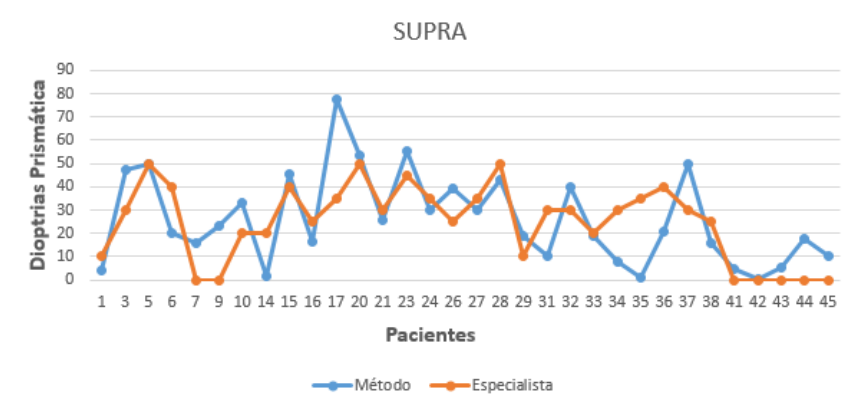

Figura 11. Resultado do método proposto na posição SUPRA em comparação com o diagnóstico do especilista.

do limbo ou a localização dos cantos dos olhos que se comparado com os demais não foram precisos. A Tabela 1 demosntra os resultados obtidos pelo método proposto.

Tabela 1. Resultados do método proposto para as cinco posições do olhar.

\begin{tabular}{|c|c|c|c|}
\cline { 2 - 4 } \multicolumn{1}{c|}{} & Sensibilidade & Espeficifidade & Acurácia \\
\hline DEXTRO & $70,4 \%$ & $40,0 \%$ & $65,6 \%$ \\
\hline INFRA & $63,0 \%$ & $40,0 \%$ & $59,4 \%$ \\
\hline LEVO & $51,9 \%$ & $40,0 \%$ & $50,0 \%$ \\
\hline PPO & $66,7 \%$ & $60,0 \%$ & $65,6 \%$ \\
\hline SUPRA & $51,9 \%$ & $80,0 \%$ & $56,3 \%$ \\
\hline
\end{tabular}

A partir dos resultados encontrados na Tabela 1 pode-se afirmar que nas posições DEXTRO e INFRA o método obteve um melhor resultado na idenficação dos pacientes estrábicos, no entanto erra ao identificar os pacientes que não possuem a potologia, devido a inclinação da região dos olhos do paciente na aquisição das imagens. Entretanto, a posição PPO alcançou o melhor resultado se comparado com os demais, pois há um equilíbrio entre as três métricas.

A Tabela 2 apresenta uma comparação dos resultados encontrados neste trabalho com alguns trabalhos relacionados. Nosso método apresenta um resultado superior ao trabalho de Loudon et al. (2011) em relação a identificação da patologia. Nosso trabalho apresenta um resultado inferior ao trabalho de Almeida et al. (2012), porém seus resultados são expressos em pixels. Os trabalhos de Lu et al. (2018) e Chen et al. (2018) apresentam um resultado superior ao nosso método, no entanto ambos utilizam redes neurais convolucionais na etapa de classificação do estrabismo. 
Tabela 2. Comparação método proposto e trabalhos relacionados.

\begin{tabular}{|c|c|c|c|}
\hline Trabalhos & Sensibilidade & Especificidade & Acurácia \\
\hline Loundon el al. (2011) & $60 \%$ & $96 \%$ & - \\
\hline Almeida et al. (2012) & $100 \%$ & $91,3 \%$ & $94 \%$ \\
\hline Lu et al. (2018) & $93,3 \%$ & $96,1 \%$ & $93,8 \%$ \\
\hline Chen et al. (2018) & $96 \%$ & $94,1 \%$ & $95,2 \%$ \\
\hline Método Proposto & $66,7 \%$ & $60 \%$ & $65,6 \%$ \\
\hline
\end{tabular}

\section{CONCLUSÃO}

Este trabalho apresentou um novo método para detectar automaticamente o estrabismo avaliando o posicionamento do centro do limbo em relação ao centro dos cantos dos olhos. Apesar de não ser um método convencional utilizado pelos especialistas, pode-se afirmar que o método apresenta um desempenho promissor para detecção do estrabismo não apenas em imagens do teste de Hirschberg como também em outros exames, podendo ser útil para a realização de triagens de pacientes.

Considerando a tolerância ao erro de $\pm 10 \Delta$, o melhor resultado encontrado foi na posição PPO obtendo sensilidade de $66,7 \%$, especificidade de $60 \%$ e acurácia de $65,6 \%$. Observa-se também que a etapa de localização precisa dos cantos dos olhos e do limbo são imprescindíveis para a detecção e quantificação correta do desvio. Portanto, pretende-se melhorar essas etapas para otimizar os resultados e utilizar outras bases de imagens de outros tipos de exames para constatar a eficácia da metodologia.

\section{AGRADECIMENTOS}

Os autores agradecem a Coordenação de Aperfeiçoamento de Pessoal de Nível Superior (CAPES).

\section{REFERÊNCIAS}

Almeida, J.D.S.d., Silva, A.C., De Paiva, A.C., and Teixeira, J.A.M. (2012). Computational methodology for automatic detection of strabismus in digital images through hirschberg test. Computers in biology and medicine, 42(1), 135-146.

ALMEIDA, J.D.S.d. et al. (2013). Metodologia computacional para detecção e diagnóstico automáticos e planejamento cirúrgico do estrabismo.

Bicas, H.E. (2009). Estrabismos: da teoria à prática, dos conceitos às suas operacionalizações. Arquivos Brasileiros de Oftalmologia, 72(5).

Chen, Z., Fu, H., Lo, W.L., and Chi, Z. (2018). Strabismus recognition using eye-tracking data and convolutional neural networks. Journal of healthcare engineering, 2018.

Choi, R.Y. and Kushner, B.J. (1998). The accuracy of experienced strabismologists using the hirschberg and krimsky tests. Ophthalmology, 105(7), 1301-1306.

Das, A., Pal, U., Ballester, M.A.F., and Blumenstein, M. (2014). Multi-angle based lively sclera biometrics at a distance. In 2014 IEEE Symposium on Computational Intelligence in Biometrics and Identity Management (CIBIM), 22-29. IEEE.

De Almeida, J.D.S., Silva, A.C., Teixeira, J.A.M., Paiva, A.C., and Gattass, M. (2015). Computer-aided methodology for syndromic strabismus diagnosis. Journal of digital imaging, 28(4), 462-473.
Dice, L.R. (1945). Measures of the amount of ecologic association between species. Ecology, 26(3), 297-302.

Hasebe, S., Ohtsuki, H., Kono, R., and Nakahira, Y. (1998). Biometric confirmation of the hirschberg ratio in strabismic children. Investigative ophthalmology \&6 visual science, 39(13), 2782-2785.

Khng, C. and Osher, R.H. (2008). Evaluation of the relationship between corneal diameter and lens diameter. Journal of Cataract \& Refractive Surgery, 34(3), 475479.

Maheshan, M., Harish, B., and Nagadarshan, N. (2018). On the use of image enhancement technique towards robust sclera segmentation. Procedia computer science, 143, 466-473.

Proença, H. and Alexandre, L.A. (2005). Ubiris: A noisy iris image database. In International Conference on Image Analysis and Processing, 970-977. Springer.

Redmon, J., Divvala, S., Girshick, R., and Farhadi, A. (2016). You only look once: Unified, real-time object detection. In Proceedings of the IEEE conference on computer vision and pattern recognition, 779-788.

Redmon, J. and Farhadi, A. (2018). Yolov3: An incremental improvement. arXiv preprint arXiv:1804.0276\%.

Ronneberger, O., Fischer, P., and Brox, T. (2015). U-net: Convolutional networks for biomedical image segmentation. In International Conference on Medical image computing and computer-assisted intervention, 234-241. Springer.

Schwartz, G.S. (2006). The eye exam: a complete guide. Slack Incorporated.

SILVA, I.F.S.d. et al. (2019). Detecção automática da presença de patologia na visão baseada em imagens do teste de brückner.

Silva, I.F., Almeida, J.D., Teixeira, J.A., Junior, G.B., and de Paiva, A.C. (2018). Teste automático de brückner basedo em imagens. In $18^{\circ}$ Simpósio Brasileiro de Computação Aplicada à Saúde (SBCAS 2018), volume 18. SBC.

Zheng, Y., Fu, H., Li, B., and Lo, W.L. (2018). An automatic stimulus and synchronous tracking system for strabismus assessment based on cover test. In 2018 International Conference on Intelligent Informatics and Biomedical Sciences (ICIIBMS), volume 3, 123-127. IEEE. 